received intubation, inotropes, or $\geq 3$ fluid boluses within one hour of transfer.

Results The average rate of ETs per 10,000 patient days decreased from 1.57 to 0.49 during the study period (figure 2). This coincided with special cause improvement in all process measures, including earlier recognition of potentially deteriorating patients and increased exemplary utilization of SA tools (figure 3).

Conclusions An innovative, proactive, and reliable process to predict, prevent, and respond to clinical deterioration was associated with a nearly $70 \%$ reduction in ETs. Importantly, ETs are associated with increased hospital length of stay and mortality.

\section{IMPLEMENTING TEAM BASED CARE TO IMPROVE CERVICAL CANCER SCREENING RATE IN COMMUNITY BASED RESIDENT RUN CLINIC}

Momena Sohail, Fatima Fayyaz, Sarah Finlay, Afnan Ammar. Trinity Health, USA

\subsection{6/bmjoq-2020-IHI.21}

Background In 2015, for every 100,000 women, 8 new Cervical Cancer cases were reported and 2 died of cancer. The introduction of effective screening has led to a steady decrease in Invasive Cervical Cancer incidence and mortality in highincome countries however Socioeconomic status and access to health have created a cervical cancer disparity gap. This QI project was undertaken to evaluate and improve cervical cancer screening rates at a community-based resident-run IM clinic.

Objectives Increase screening rate to $50 \%$ by Dec 2018 and sustain increase rates to above $45 \%$ in all months in 2019.

Methods At the start of 2018, physician data reported that screening rates at the clinic were $36 \%$, lower than those of our other clinical practices. A clinical survey was completed to assess patient understanding of cervical cancer screening. The first intervention was sharing individual provider data with the residents. This was a weak intervention and did not improve rates of screening. Our second intervention was a team-based approach and pre-visit planning: identify patients and calling them1-2 days ahead and including PAP in the daily huddle.

Results The original survey showed that out of 124 women, 66 needed screening. Surveys intensified lack of continuity; several listed PCPs were not seeing their own patients, patients unsure of resources, and financial concerns as barriers. The team-based approach showed that more PAPs were being done per week, and was streamlined with staff feedback to minimize disruption to current workflow. With a goal of $50 \%$ by the end of 2018 , we were able to achieve $48.9 \%$ screening rates. The increase was sustained above $45 \%$ in $8 / 12$ months in 2019 (figure 1).

Conclusions Team-based practice is an effective practice in increasing cervical cancer screening to overcome challenges in continuity of care, health access disparity, and resource allocation in a primary care clinic.

\section{IMPLEMENTATION OF A PHARMACIST-DRIVEN ANTIMICROBIAL TIME-OUT FOR MEDICAL-SURGERY SERVICES IN AN ACADEMIC PEDIATRIC HOSPITAL}

Christopher Stang ${ }^{1}$, Jessica Tansmore ${ }^{1}$, Katelyn Parson", Kathryn Nuss ${ }^{1}$, Matthew Sapko ${ }^{1}$ Zachary Thompson", Don Buckingham1, Mahmoud Abdel-Rasoul', Joshua Watson", Preeti Jaggi ${ }^{2}$. 'Nationwide Children's Hospital, United States; ${ }^{2}$ Children's Healthcare of Atlanta, United States

\subsection{6/bmjoq-2020-IHI.22}

Background This quality improvement initiative implemented a pharmacist-driven antimicrobial time-out (ATO) in a large, free-standing pediatric hospital.

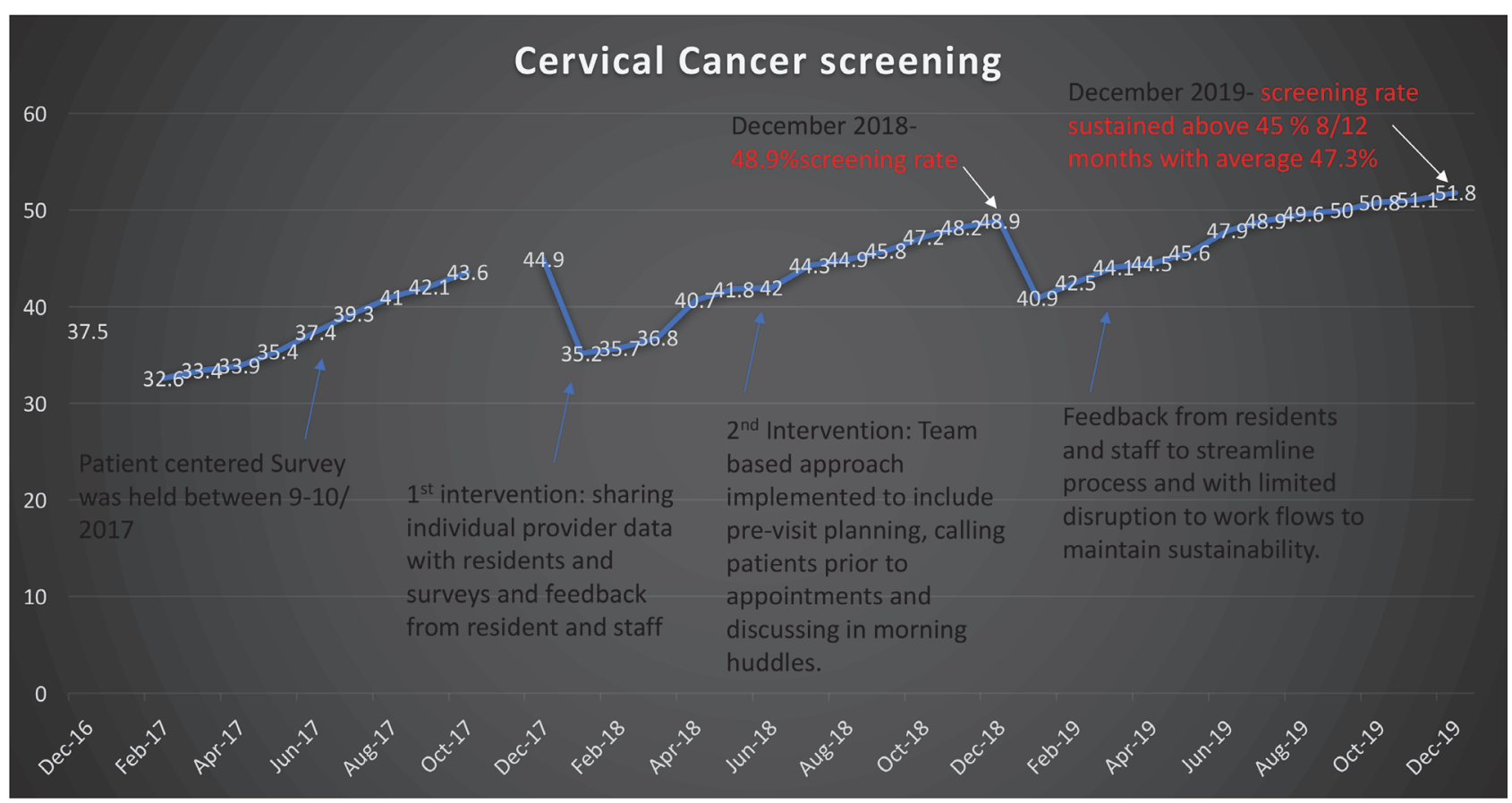

Abstract 21 Figure 1 